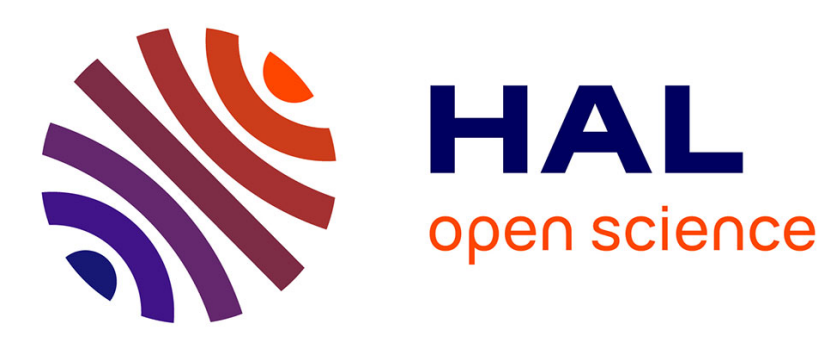

\title{
Le Prince et la question constitutionnelle
}

Jérémie Barthas

\section{To cite this version:}

Jérémie Barthas. Le Prince et la question constitutionnelle. La Pensée, 2021, Machiavel. Le peuple, la politique, l'expérience, 406, 10.3917/lp.406.0048 . hal-03506528

\section{HAL Id: hal-03506528 \\ https://hal.science/hal-03506528}

Submitted on 2 Jan 2022

HAL is a multi-disciplinary open access archive for the deposit and dissemination of scientific research documents, whether they are published or not. The documents may come from teaching and research institutions in France or abroad, or from public or private research centers.
L'archive ouverte pluridisciplinaire HAL, est destinée au dépôt et à la diffusion de documents scientifiques de niveau recherche, publiés ou non, émanant des établissements d'enseignement et de recherche français ou étrangers, des laboratoires publics ou privés. 
Le Prince et la question constitutionnelle.

(version acceptée, pour La pensée, $n^{\circ} 406$, juin 2021)

1. Un important succès de la critique textuelle et de l'histoire intellectuelle concerne un ouvrage du canon de l'enseignement de théorie politique en Angleterre : les Two Treatises of Government de John Locke, dont l'historien Peter Laslett parvint à démontrer que l'histoire rédactionnelle n'était pas liée à la révolution de 1688 - ainsi qu'on l'avait admis jusque-là -, mais à des circonstances politiques et littéraires antérieures de presque une décennie. Par conséquent, les deux traités politiques que Locke réunit et publia en 1689 ne pouvaient plus être interprétés comme une rationalisation d'une révolution ayant besoin d'une défense théorique.

Ce succès illustre combien les questions historiques les plus anodines en apparence, comme celles de datation et de chronologie, ne sont pas forcément celles qui trouvent facilement une réponse adéquate. Alors qu'il est généralement admis que les ouvrages de théorie politique comportent une dimension pratique d'intervention dans les conflits de leur temps, on peut s'éloigner fortement du chemin de la connaissance si, pour un texte donné, la chronologie des faits qui lui sont relatifs n'est pas établie d'une façon qui permette d'identifier d'importants composants du problème que la théorie s'emploie justement à résoudre.

Cette insuffisance peut avoir pour cause l'apparente imprécision des éléments internes au texte, le manque d'évidences externes ou la méconnaissance de celles qui sont accessibles, parfois à cause d'œillères disciplinaires. La carence d'information ouvre alors à une multiplicité de schémas interprétatifs concurrents, du plus sérieux au plus fantaisiste, et qui peuvent donner le sentiment de s'annuler les uns les autres. Une information plus complète, au contraire, permet de réduire significativement les marges de l'erreur interprétative. Elle contribue à l'occasion à bouleverser en profondeur les approches et l'évaluation d'un texte donné, comme cela a été le cas avec l'édition critique du traité politique de Locke par Peter Laslett ${ }^{1}$.

Quant à lui, Le Prince de Machiavel reste après cinq siècles l'objet d'un conflit d'interprétation tout à fait saisissant au sujet de son histoire rédactionnelle. Il y a environ un an, j'ai eu I'honneur de faire paraître dans la Rivista Storica Italiana une étude assez détaillée

\footnotetext{
${ }^{1}$ 1re éd., Cambridge, CUP, 1960.
} 
à ce propos, offrant en annexe un document inédit conservé aux Archives de Florence ${ }^{2}$. Ce document est une Constitution, c'est-à-dire un texte organisant la division et les relations entre ce que nous appellerions aujourd'hui les (plus hauts) organes de l'État. Cette Constitution a été validée par les autorités florentines compétentes le 22 novembre 1513 et certaines de ses dispositions ont été appliquées dès le 15 décembre. C'est entre ces deux dates que Machiavel a annoncé avoir rédigé Le Prince. Pour I'histoire politique et institutionnelle de Florence, l'importance de l'acte du 22 novembre 1513 est évidente : il pose la loi fondamentale de la République florentine sous régime médicéen jusqu'à la révolution de mai 1527, qui l'annula et réintroduisit pour trois années le régime démocratique du Grand Conseil renversé en septembre 1512. Pourtant, ce texte constitutionnel est resté à l'écart des analyses de la «conscience politique» de cette période de transition ${ }^{3}$ et, plus particulièrement, du débat sur l'histoire rédactionnelle et la signification du Prince de Machiavel.

2. Ce débat a été relancé en 1980 par une tentative de réviser en profondeur l'hypothèse de datation la mieux admise. Selon cette dernière, Le Prince aurait été composé d'un jet, durant l'été et l'automne 1513, se rattachant aux desseins que Léon X (Giovanni de' Medici) pouvait avoir pour son frère puîné, Giuliano, ou pour le fils de son frère aîné, Lorenzo, en matière d'affaires intérieures et extérieures ${ }^{5}$. D'après l'hypothèse révisionniste, en revanche, le texte aurait fait l'objet d'au moins deux rédactions bien distinctes, la première en pensant à Giuliano et les suivantes à Lorenzo ; la date de 1513 vaudrait pour la première rédaction, tandis que l'état du texte qui nous est parvenu, avec sa dédicace à Lorenzo, pourrait dater de la fin de l'année 1518. Selon cette version radicale de l'hypothèse révisionniste, Machiavel se serait alors trouvé impliqué dans un complot visant, contre l'avis de Léon X, à faire de Lorenzo le maître absolu de Florence et à renverser définitivement ce qui restait des institutions

\footnotetext{
2 J. Barthas, « La composizione del Principe di Machiavelli e la restaurazione dei Medici a Firenze ", Rivista Storica Italiana, 131, n 3 (Déc. 2019), p. 761-811.

${ }^{3}$ Cf. R. Von Albertini, Das florentinische Staatsbewusstsein im Übergang von der Republik zum Prinzipat, Berne, Francke, 1955.

${ }^{4} \mathrm{~N}$ 'a pas suffi à l'y introduire la mention - indirecte - in N. Machiavelli, /l Principe, nouv. éd. G. Inglese, Turin, Einaudi, 1995, p. xxv note 2.

${ }^{5}$ Cf. F. Chabod, "Sulla composizione de I/ Principe di Niccolò Machiavelli » (1927), in Id., Scritti su Machiavelli, Turin, Einaudi, 1964, p. 139-93.
} 
républicaines. Il aurait ainsi soumis un traité d'abord technique - sur la prise du pouvoir et sa conservation - à un profond remaniement, afin qu'il puisse appuyer sur le plan culturel un putsch préparé par Lorenzo ${ }^{6}$. Le Prince devrait donc être interprété comme une rationalisation anticipée d'un coup d'État qui aurait eu besoin d'une défense théorique si Lorenzo était parvenu à le réaliser.

Entre répliques et contre-répliques, répétitions, formulations d'hypothèses intermédiaires, synthèses des débats et tentatives de conciliation, le travail de cette théorie originale - et incroyablement compatible avec la notion que véhicule l'inquiétant nom de Machiavel dans le langage courant - a formé une partie impressionnante de la littérature spécialisée. Depuis l'édition critique de référence, publiée en 1994 par Giorgio Inglese, des points d'interprétation particuliers ont pu être affinés et des équivoques levées ${ }^{7}$. S'est aussi dessinée une représentation plus précise de l'archétype dont dépend la première division de la tradition manuscrite : cet archétype apparaît aujourd'hui comme la « transcription (pas même très scrupuleuse) d'un autographe écrit d'un jet [...] et qui avait l'état d'une minute ", porteuse d'écritures « interlinéaires ou dans les marges », ce qui a pu faciliter au moment de la copie quelques erreurs d'insertion plus ou moins problématiques ${ }^{8}$.

Sur le fond, l'hypothèse traditionnelle est sortie renforcée de ce débat, tous les arguments construits par les révisionnistes pour illustrer la thèse des multiples rédactions ayant été démontés. Pour autant, dans le cadre de l'hypothèse traditionnelle, la question de la date de la dédicace à Lorenzo n'a pas encore trouvé une réponse qui permette d'intégrer de manière cohérente la totalité des faits connus et des inductions les plus probables. Une ultime conjecture s'arrête au printemps 1514 et envisage la possibilité que le texte ait été consigné à ce moment-là ${ }^{9}$, mais elle se fonde sur une hypothèse récusable de datation d'un fragment autographe où Machiavel décrit le comportement « civil » de Lorenzo à l'égard de ses concitoyens. Cette situation pourrait laisser penser qu'il reste une once de négociable dans l'hypothèse révisionniste. De fait, les partisans de cette dernière - qui s'est affichée dans la

\footnotetext{
${ }^{6}$ Cf. M. Martelli, « Da Poliziano a Machiavelli. Sull'epigramma Dell'Occasione e sull'occasione », Interpres, 1 (1979 [oct. 1980]), p. 230-54, et l'introd. de son édition du Principe (Ed. Nazionale, Rome, Salerno, 2006). ${ }^{7}$ Cf. N. Machiavelli, De Principatibus, éd. crit. G. Inglese, Rome, ISIME, 1994, et Id., II Principe, nouv. éd. G. Inglese, Turin, Einaudi, 2013.

${ }^{8}$ G. Inglese, «Sul testo del Principe », La Cultura, 51, n 1 (avr. 2014), p. 47-76: p. 72. Sur une (probable) erreur d'insertion dans le chap. 3, cf. Barthas, " La composizione ", append. 2.

${ }^{9}$ Cf. l'introd. de l'éd. Inglese 2013, p. XIX-XXX, en partic. p. XXV-XXVI.
} 
monumentale Edizione nazionale delle opere di Machiavelli - ont fini par reconnaître qu'elle tenait surtout de l'ordre de la conviction et que, en somme, il fallait y croire même si les faits matériels connus et la tradition manuscrite n'en donnaient pas la moindre preuve et conduisaient à des conclusions contraires ${ }^{10}$.

3. Rappelons donc les données essentielles, et en premier lieu celles auxquelles un lecteur prenant au sérieux la question chronologique a déjà accès en consultant les deux premières éditions.

a) L'édition princeps du Principe a été imprimée à titre posthume, en janvier 1532 à Rome, sous les auspices du pape Clément VII (Giulio de' Medici) et dans le cadre officiel d'une politique culturelle de valorisation de la langue toscane et d'accompagnement des Médicis dans leur marche vers l'absolutisme. Fin mai 1532, quelques semaines après l'avènement d'Alessandro de' Medici comme premier «Duc de la République florentine ", l'éditeur florentin Bernardo Giunta contestera cette appropriation, responsable d'un anachronisme interprétatif ternissant déjà la mémoire de Machiavel.

b) Giunta précise aussi que le traité a déjà fait l'objet d'une contrefaçon latine, faisant allusion à L'Art de régner d'Agostino Nifo de' Medici (imprimé en 1523).

c) Le traité porte une lettre de dédicace sans date " al Magnifico Lorenzo di Piero de' Medici » qui mourut dans sa vingt-sixième année, en mai 1519.

d) Dans le chapitre 26, l'opportunité historique du dédicataire - son " occasion » - est associée aux espérances suscitées par l'élection et l'intronisation de Giovanni de' Medici à la tête de l’Église (Pr. 26.8) $)^{11}$, en mars-avril 1513.

e) L'évènement le plus récent évoqué dans Le Prince figure dans ce même dernier chapitre : il s'agit d'un fait de guerre advenu début octobre 1513 (Pr. 26.19). Aucune des analyses du rapport de puissances ne prend en compte des configurations postérieures à cette date. Par exemple, la situation de grande fragilité de la France dont il est question dans le chapitre 13 vaut pour l'été et l'automne 1513, quand les troupes anglaises et impériales étaient présentes sur son territoire (Pr. 13.19).

\footnotetext{
${ }^{10}$ Cf. F. Bausi, /l Principe, dallo scrittoio alla stampa, Pise, Ed. della Normale, 2015, p. 13.

${ }^{11}$ Pour le texte du Principe (Pr.), l'éd. Inglese 1994 a fixé la numérotation en segment - adoptée notamment par J.-L. Fournel et J.-Cl. Zancarini dans leur trad. (2de éd., Paris, PUF, 2014).
} 
Ces éléments permettaient de distinguer l'histoire rédactionnelle de l'histoire éditoriale : cette dernière s'inscrivait dans la phase initiale du passage effectif, et encore alors contesté, de Florence de la république au principat (héréditaire) médicéen, mais interpréter Le Prince comme une rationalisation de ce passage, tient d'une application rétrospective douteuse. De plus, la contrefaçon de Nifo démontre que Le Prince connut une circulation manuscrite avant 1523 et que le pouvoir médicéen en tenait la substance politique pour suspecte. Surtout, le présent politique du texte se situe dans une période antérieure : plus précisément, une période qui va du printemps 1513 à l'automne de cette même année.

Cette perception raisonnable a été à la fois confirmée et compliquée par la publication, en 1810, de l'une des plus célèbres lettres de la littérature italienne : celle que Machiavel adresse le 10 décembre 1513 à Francesco Vettori, alors ambassadeur-résidant à Rome. Machiavel y déclare plusieurs choses:

a) qu'il a développé une " science » de l'action des hommes d’État, récemment consignée dans un court traité intitulé De Principatibus ;

b) qu'il dédicace ce traité à Giuliano de' Medici - lequel frayait alors à Rome, dans l'entourage de Léon $\mathrm{X}$ - en vue de lui offrir ses services ;

c) qu'il en a déjà donné à lire le manuscrit à un ami commun - Filippo Casavecchia - qui, s'étant rendu à Rome, pourra parler du contenu du texte à Vettori ;

d) qu'il est en train de réviser ce manuscrit ;

e) qu'il s'est interrogé avec Casavecchia sur le choix du dédicataire, ainsi que sur l’opportunité et la façon de lui présenter l'ouvrage. Sur ces points, Machiavel demande expressément conseil à Vettori, susceptible de servir d'intermédiaire.

La lettre du 10 décembre 1513 est donc cause que l'histoire rédactionnelle du Prince est devenue problématique, dans la mesure où l'ouvrage nous est parvenu avec une dédicace non datée adressée au jeune Lorenzo, et non pas à Giuliano.

4. Les chercheurs se sont ainsi demandé quand fut rédigée la dédicace à Lorenzo et dans quelle mesure le texte du traité évoqué le 10 décembre 1513 différait de celui que nous connaissons. Ils se sont divisés quant aux réponses à apporter, mais ils ont élaboré leurs différentes hypothèses en travaillant dans le cadre d'un seul et unique paradigme. Celui-ci repose sur l'idée que Machiavel avait d'abord le projet d'approcher Giuliano et qu'il ne se décida en faveur de Lorenzo qu'en seconde intention. Pour certains, Machiavel aurait pris ce parti entre 
février et mai 1514, probablement après avoir compris que Vettori se refusait à jouer son rôle d'intercesseur dans ce cas; pour d'autres, ce choix ne saurait être antérieur au 31 janvier 1515.

Les sources sur lesquelles se fondent ces différentes positions sont essentiellement la correspondance entre Machiavel et Vettori, qui court du 13 mars 1513 au 31 janvier 1515, et la tradition manuscrite du Prince, dont les premiers témoins restent de datation incertaine. De l'étude de cette dernière, il résulte que l'archétype, et donc le manuscrit original qui le précède, est antérieur à la mort du roi de France Louis XII. En effet, celui-ci est donné vivant dans l'une des deux branches de la tradition, tandis que l'autre est manifestement corrompue sur ce point, par effet d'une mise à jour maladroite (en Pr. 16.8-9). Or, ces deux branches - qui ne présentent pas de variantes justifiant la théorie des rédactions multiples - comportent la dédicace à Lorenzo. Par conséquent, il paraît extrêmement difficile de concéder que cette dernière puisse être postérieure à la mort de Louis XII, le 31 décembre 1514.

Dans la correspondance Machiavel-Vettori, l'ouvrage annoncé le 10 décembre 1513 n'est plus mentionné après le 18 janvier 1514. Il est douteux que Vettori en ait vu le manuscrit. En effet, en décembre 1514 et janvier 1515, Machiavel ne présuppose pas que celui-ci ait eu connaissance des analyses qu'il y a développées, notamment sur les rapports entre puissances européennes et la place de la monarchie pontificale, ou sur les difficultés que présente la normalisation d'un nouveau régime. Or, le 31 janvier 1515, il apparaît que Machiavel avait encore les yeux tournés vers Giuliano, alors en passe de former un État nouveau dans le Nord de l'Italie. Même si l'expression d'une offre de service est absente de cette lettre ${ }^{12}$, cela a pu suggérer l'idée qu'il n'avait pas encore abandonné l'idée de lui dédier son traité.

Tel est donc le point où frotte l'interprétation la plus plausible des origines de la tradition manuscrite. Le nom de Lorenzo n'apparait jamais lié au Prince dans la correspondance Machiavel-Vettori, contrairement à celui de Giuliano, qui l'est explicitement le 10 décembre 1513 et le demeure implicitement le 31 janvier 1515, où Machiavel insiste comme dans les chapitres 7 et 17 de son traité - sur l'exemplarité de la figure de César Borgia. C'est pourquoi les partisans de l'hypothèse révisionniste tiennent à cette lettre comme à leur dernière ligne de défense et qu'ils s'efforcent de maintenir l'idée d'un remaniement du

${ }^{12}$ Cf. J. M. Najemy, Between Friends. Discourses of Power and Desire in the Machiavelli-Vettori Letters of 1513-1515, Princeton, Princeton Univ. Press, 1993, p. 330. 
chapitre 7 postérieur à janvier 1515 et effectué dans la perspective d'adresser le traité à Lorenzo ${ }^{13}$. Les révisionnistes procèdent à la manière d'un archéologue qui trouverait une poterie complète, mais la casserait pour s'attacher à en dater séparément les morceaux sans plus parvenir à les recoller. La procédure est en elle-même discutable, mais c'est en fait le paradigme même de la dédicace première à Giuliano qui trouve ici son point de rupture. Si, comme l'étude de la tradition manuscrite a permis de l'établir, la dédicace à Lorenzo était présente avant le 31 décembre 1514, et si, comme l'analyse de la correspondance MachiavelVettori le laisse penser, l'auteur du Prince eut en vue Giuliano entre le 10 décembre 1513 et le 31 janvier 1515, alors il faut conclure que la dédicace à Lorenzo devait être antérieure aux annonces faites le 10 décembre 1513.

5. Avancer l'idée que la dédicace à Lorenzo précédait l'hypothèse d'une dédicace à Giuliano, revient donc à proposer un changement de paradigme dont il faudra tirer toutes les implications pour évaluer Le Prince dans sa dimension pratique de texte d'intervention. J'indiquerai ci-après l'une d'entre-elles, la principale à mon sens. Mais il faut d'abord relever que rien ne s'oppose actuellement à la formation de ce nouveau paradigme. II permet en revanche de mieux situer d'autres écrits de Machiavel : par exemple, le texte en rime appelé Pastorale, qui se termine par l'annonce d'une œuvre plus ambitieuse, fut vraisemblablement écrit en hommage à Lorenzo juste après son arrivée au pouvoir ${ }^{14} ; s^{\prime} y$ rattacherait ce fragment autographe où Machiavel témoigne de l'attitude " civile » et des progrès rapides de Lorenzo au moment de son installation ${ }^{15}$. Des considérations historiques plus générales contribuent aussi à renforcer les fondements de ce nouveau paradigme.

Après avoir été innocenté de toute participation à la conjuration contre les Médicis de février 1513, Machiavel avait exprimé l'espoir que les nouveaux patrons de Florence levassent aussi les autres peines prononcées contre lui cinq mois plus tôt. En effet, sa relégation l'empêchait de prétendre à un emploi public jusqu'au 17 novembre 1513. Or, depuis le départ du cardinal Giovanni pour le conclave et son accession au trône pontifical, Giuliano dirigeait le gouvernement provisoire de la République florentine et Machiavel voulait croire que ce

\footnotetext{
${ }^{13} \mathrm{Cf}$. P. Larivaille dans l'introd. de sa trad. du Prince suivi de L'Art de régner d'A. Nifo (Paris, Belles lettres, 2008), p. XLIX.

${ }^{14}$ Cf. mon c. r. du Giuliano de' Medici de J. Jungić (2018), in Riv. Stor. It., 131, nº 2 (sept. 2019), p. 713-19.

${ }^{15} \mathrm{Cf}$. Barthas, « La composizione », p. 774 note 56.
} 
dernier lui était favorable. II s'avéra vite que la situation personnelle de l'ex-chancelier n'entrait pas dans les préoccupations de Giuliano. La relégation ne fut pas levée. Le 17 novembre 1513 resta l'horizon d'attente de Machiavel.

Parallèlement, le jeune Lorenzo avait été coopté au sein du gouvernement provisoire (la Balía), par dérogation d'âge. Son rôle politique se renforça rapidement. Dès la mi-juin 1513, Léon X s'accorda avec les ambassadeurs florentins pour le désigner chef de la ville, au détriment de Giuliano qui avait sa préférence. Dans une société obsédée par les questions de parentèle, la logique lignagère était favorable à l'aîné des mâles en ligne directe de la branche de la famille chassée par les Florentins à l'automne 1494. Une loi d'avril 1492 avait déclaré Piero di Lorenzo de' Medici " premier homme de la cité ${ }^{16}$; à partir d'août 1513 , son fils Lorenzo fut placé dans une position similaire, de facto et de jure, c'est-à-dire celle d'un " prince " au sens de premier membre par préséance au sein du gouvernement provisoire, à la fois garant de son autorité et guide de ses décisions, par analogie relative avec la figure du princeps senatus sous la République romaine.

Machiavel forma le projet du Prince avec l'espoir que les nouveaux patrons de Florence reconnaissent ses qualités d'analyse et de synthèse des problématiques rencontrées par tous les États, et plus particulièrement par les principats « récents ». En juillet 1513, Vettori avait indiqué à Machiavel l'intention de Léon X de donner certains États « aux siens, c'est-à-dire à Giuliano et Lorenzo ». Lorsque Machiavel mit en œuvre son projet et fit le choix d'un dédicataire, la logique lignagère qui s'était imposée au pape devait s'imposer à lui aussi. L'enfreindre serait revenu à prendre d'emblée un parti inutilement risqué dans une querelle de préséance. Il aurait fallu une excellente raison pour le faire.

Or il n'y en avait pas lorsque Lorenzo arriva à Florence et remplaça officiellement Giuliano, avec pour objectif prioritaire la normalisation du régime né du coup d'État de 1512. La sortie du gouvernement provisoire et de l'état d'exception par l'introduction d'un nouvel ordre constitutionnel était très attendue dans l'opinion publique ; les sources contemporaines indiquent même que Lorenzo reçut pour cela un accueil favorable ${ }^{17}$. La dédicace du Principe exprime ce sentiment général lorsque sont mises en avant les promesses dont étaient porteuses la fortune et les autres qualités du nouveau prince. Parmi celles-ci, Machiavel met

\footnotetext{
${ }^{16}$ Cit. in N. Rubinstein, The Government of Florence under the Medici (1434-1494), 2nde éd., Oxford, Clarendon, 1997, p. 252.

${ }^{17}$ Cf. B. Cerretani, Ricordi, éd. G. Berti, Florence, Olschki, 1993, p. 310.
} 
en avant la jeunesse. En effet, lorsqu'il exalte la fougue et l'audace des jeunes, à la fin du chapitre 25 , il le fait en des termes appropriés pour Lorenzo, et non pour Giuliano de treize ans son aîné. Après avoir analysé les causes de la crise de l'Italie (Pr. 24), Machiavel présente cette crise comme une occasion extraordinaire pour un jeune homme audacieux que la fortune a mis en position de prouver sa virtù (Pr. 25), avant d'énoncer un programme de politique générale se réclamant des plus puissants mythes de libérateurs et de fondateurs (Pr. 26).

6. L'idée d'une dédicace première à Giuliano s'est formée en tirant une conclusion nonnécessaire et abusive de la lettre à Vettori du 10 décembre 1513. Pour son destinataire, le fait que Machiavel y nomme Giuliano devait plutôt apparaître comme une renonciation tacite à Lorenzo, seul des deux à occuper la position d'un « prince » récemment constitué. Cela peut contribuer à expliquer la circonspection de Vettori à l'égard de la demande de Machiavel. Dès lors, la question centrale est la suivante : pourquoi, dans les jours qui précèdent la rédaction de cette lettre, Machiavel aurait-il donc envisagé de renoncer à dédicacer son traité à Lorenzo?

Pour résoudre les questions touchant la chronologie du Prince, une tendance récente parmi les historiens consiste à chercher une réponse dans la vie privée de l'auteur ${ }^{18}$. L'opération n'a pas été concluante jusqu'ici et elle a l'inconvénient de rester à l'extérieur de la substance théorique et politique du texte. Un plus grand bénéfice interprétatif était jadis attendu de la détermination d'une date plafond, c'est-à-dire de l'identification d'un fait historique qui aurait dû avoir un impact sur la physionomie du texte : par exemple, le dernier chapitre du Prince ignore la loi sur le recrutement militaire du 19 mai 1514, alors même que Machiavel y appelle à rétablir un programme de conscription (Pr. 26.20-21) ${ }^{19}$.

Maintenir que le présent politique du Prince se situe entre le printemps et l'automne 1513, et ajouter à cela que c'est cette période qui doit être qualifiée pour saisir l'intention dont est porteuse la dédicace à Lorenzo, commande de rechercher le fait élémentaire décisif pour la réalisation duquel le jeune chef de Florence eut un rôle particulier et qui entrait suffisamment en contradiction avec la substance de la pensée développée dans Le Prince pour

\footnotetext{
${ }^{18}$ Cf. W. J. Connell, « Dating The Prince », Review of Politics, 75, n 4 (oct. 2013), p. 497-514.

${ }^{19}$ Cf. G. Sasso, "II Principe ebbe due redazioni ? " (1981), in Id., Machiavelli e gli antichi e altri saggi, vol. 2, Milan-Naples, Ricciardi, 1988, p. 197-276, en partic. p. 206-08.
} 
conduire son auteur à remettre en question sa volonté initiale de le lui adresser. Or, ce fait apparait clairement à la lecture des manuscrits des actes du gouvernement provisoire, c'està-dire de la Balia établie le 16 septembre $1512^{20}$.

L'instauration de la Balia avait résulté du coup d'État aristocratique du 31 août 1512 ayant réintroduit à Florence les héritiers de Laurent le Magnifique après dix-huit ans d'exil. Depuis lors, la capitale de la Toscane avait été gouvernée par cette commission extraordinaire d'une soixantaine de membre, dotée de prérogatives très étendues, en principe pour une année. Ses pouvoirs incluaient en particulier celui d'élaborer et d'établir une nouvelle constitution. Une émanation plus réduite de cette commission avait d'abord travaillé en ce sens sous la direction de Giovanni de' Medici, mais son élection au pontificat entraina à Florence une crise de leadership qui laissa la réforme constitutionnelle en suspens. Peu après l'entrée en fonction du jeune Lorenzo, mi-août 1513, la Balia fut renouvelée et le travail constitutionnel pût reprendre. Il aboutira le 22 novembre 1513 par le vote du texte voulu par Lorenzo. La contre-révolution aristocratique qui avait renversé la république populaire du Grand Conseil à la fin de l'été 1512 trouvait-là sa conclusion ${ }^{21}$.

Après un préambule, le texte constitutionnel comporte quatre titres, sous lesquels quatre organes sont définis, en spécifiant le nombre de membres, leurs qualités requises, la façon de les élire et de les remplacer, la durée de la fonction et bien sûr l'étendue de leurs pouvoirs. En fait, grâce à l'institution du premier organe, le Conseil des Soixante-dix, dont les membres (appartenant déjà pour la plupart à la Balia) devaient être indirectement choisis par Lorenzo, le nouveau régime médicéen contrôlerait pratiquement toutes les branches du système politique. Le préambule ne laisse aucun doute sur le sens de la nouvelle constitution : il s'agit d'une restauration pure et simple de l'ancien régime, ou, selon les termes qui y sont employés, d'une « recondu[ction des] choses de la ville au mode et à l'ordre de l'ancien État et gouvernement ", précédant la révolution de 1494.

7. Or, dans Le Prince, Machiavel prend position contre cette formule constitutionnelle. II le fait dans le chapitre 9, Du principat civil, où il pose qu'il existe une division irréductible entre le peuple et les grands, respectivement caractérisés comme réfractaires à l'oppression et

\footnotetext{
${ }^{20}$ Cf. Archivio di Stato di Firenze, Balíe, 43 et 44.

${ }^{21}$ Cf. R. Devonshire Jones, Francesco Vettori, Londres, Athlone, 1972, p. 83.
} 
oppresseurs. Machiavel y recourt au terme " civil » en deux sens différents : d'abord, pour évoquer une forme douce d'usurpation du pouvoir, celle qui ne se fait pas par scélératesse ou manu militari (comme dans le chapitre 8), mais avec le consensus ou des grands ou du peuple ; ensuite, pour évoquer une façon de gouverner respectueuse de la légalité et des usages, afin de mettre en évidence la difficulté que peut avoir un prince à sortir de ce cadre, en cas de nécessité, s'il n'a pas acquis le soutien du peuple ${ }^{22}$.

Bien qu'il ne nomme pas explicitement l'un ou l'autre des membres de l'illustre famille en ce chapitre, il était évident aux contemporains de Machiavel qu'il y présentait une analyse de l'expérience du pouvoir Médicéen entre 1434 et 1494, et en particulier du système politique mis en place par Laurent le Magnifique à partir de 1480. Par exemple, dans sa contrefaçon du Prince, Nifo ne le comprit pas autrement ${ }^{23}$. Plus précisément, Machiavel s'arrête dans la partie finale du chapitre 9 sur ce qui contribua à entraîner la chute du régime « civil » que le Magnifique avait de facto hérité de Cosimo et Piero di Cosimo de' Medici et qu'il avait perfectionné. Le cœur de son système était justement le Conseil des Soixante-dix, institué par la loi constitutionnelle du 19 avril 1480. Les trente membres de la Balia alors constituée sous la direction du Magnifique s'étaient dotés de l'autorité de coopter librement quarante autres citoyens éminents pour former ce nouveau corps, ayant lui-même le pouvoir de s'auto-proroger tous les cinq ans qui lui assure la pérennité. Certains documents officiels l'appellent "Sénat», d'autres évoquent "l’Ordre des Soixante-dix ». La révision constitutionnelle de 1480 validait ainsi, en la réalisant, l'idée d'une classe particulière de citoyens de rang supérieur devant être le fondement d'un conseil spécifique au sommet de la hiérarchie des pouvoirs.

Ce Sénat de type nobiliaire ou aristocratique avait une fonction consultative générale sur toutes les affaires publiques. Il flanquait les organes chargés des fonctions exécutives et ses avis devaient prendre effet auprès de ces derniers. Il était également un organe partiel de la fonction législative pour tous les textes concernant la sécurité intérieure, le système électoral, l'administration fiscale, ou le fonctionnement des autres pouvoirs constitués. De plus, il était doté d'une compétence spéciale en matière d'affaires étrangères, diplomatiques et militaires. Son fondement et l'étendue de ses prérogatives étaient tels que des intellectuels

\footnotetext{
${ }^{22}$ Cf. la note de comm. de G. Pedullà dans son éd. du Principe (Rome, Donzelli, 2013), p. 104-05 note 2.

${ }^{23}$ Cf. Nifo, De Regnandi Peritia, liv. 2, chap. 10 (éd. Larivaille, p. 232).
} 
florentins dénoncèrent immédiatement le caractère malhonnête, liberticide et tyrannique du système politique ainsi instauré ${ }^{24}$.

Dans le chapitre 9, Machiavel fait allusion à ce système en évoquant une technique de gouvernement « par le moyen des magistrats » (Pr. 9.24), car ce Sénat recrutait en son sein les membres des deux piliers du pouvoir exécutif (le bureau en charge des affaires extérieures et celui en charge de la politique financière) et ceux de la commission responsable des élections des membres de la Seigneurie et de la plupart des candidats aux offices; en 1489, ce Sénat avait même vu ses compétences législatives ultérieurement renforcées, en devenant l'organe partageant avec la Seigneurie la compétence de créer l'organe suprême de la fonction législative (le Conseil de Cent).

Après le coup d'État de 1512 , le système de gouvernement mis en place par le Magnifique se présentait à ses héritiers comme le modèle de référence vers lequel tendre. Si tôt que Giovanni partit pour Rome, Giuliano affirma à Florence une position de primus inter pares au sein de la Balía, qu'il s'employa à faire apparaître elle-même comme une forme de sénat. De son côté, Giovanni ne tarda pas à exprimer son souhait que Florence sorte du régime d'exception de la Balía, qu'il jugeait dommageable à la vie civile, et il envisageait pour cela une forme affine au système mis en place par son père ${ }^{25}$. Mais ce système n'en avait pas moins été traversé d'importantes contradictions internes. Seule la personnalité du Magnifique, au sein d'une organisation essentiellement clientéliste et mafieuse, permit de maintenir un équilibre qui devait se rompre après sa mort. La chute des Médicis advint lorsque son fils Piero fut tenté ou contraint de remettre en cause le fondement aristocratique de ce régime ${ }^{26}$.

Ainsi, le chapitre 9 du Prince enregistre la faillite de la formule politique d'une république aristocratique constituant un ordre séparé centré sur la figure d'un « prince civil » - forme florentine intermédiaire entre le « prince du Sénat » de la République romaine et le « tyran tacite et voilé » de la théorie juridique médiévale ${ }^{27}$-, telle qu'elle avait été abolie par la révolution de 1494. De ce point de vue, il y a tout lieu de retenir que, lorsqu'il rédigea ces pages, Machiavel avait bien à l'esprit celui qui était à la tête de Florence à partir de l'été 1513

\footnotetext{
${ }^{24}$ Cf. A. Rinuccini dans la continuation des Ricordi storici de F. Rinuccini, éd. G. Aiazzi, Florence, Piatti, 1840, p. CXXXII-XXXIII.

${ }^{25}$ Cf. P. Parenti, Storia fiorentina. III (1502-1518), éd. A. Matucci, Pise, Ed. della Normale, 2018, p. 431-36.

${ }^{26}$ Cf. Id., Storia fiorentina. I (1476-1478, 1492-1496), éd. A. Matucci, Florence, Olschki, 1994, p. 32.

27 Bartole, De Tyranno, chap. 12 (trad. S. Parent, Paris, Belles Lettres, 2019, p. 126 sq.).
} 
et qui devait réaliser la réforme des institutions censée normaliser le régime et stabiliser la République. Le jeune Lorenzo n'aurait pas pu lire le chapitre 9 du Prince autrement que comme un avertissement politique concernant la fragilité du système instauré par son aïeul et comme une prescription de politique intérieure. Car, si ce chapitre contient bien un enseignement prescriptif, fondé sur l'analyse du réel et de l'histoire, il repose sur un énoncé synthétique, qui est aussi un motif essentiel du Prince : faire le nécessaire pour « avoir l'amitié du peuple » (Pr. 9.18). Pour Machiavel et ses contemporains florentins, établir cette forme de partenariat impliquant des devoirs et des obligations mutuelles ne signifiait pas autre chose que de restaurer le Grand Conseil, organe de la souveraineté populaire entre 1494 et 1512. Machiavel a été tout à fait explicite sur ce point lorsqu'il a été consulté sur la réforme de la Constitution de novembre 1513 après la mort du dédicataire du Prince ${ }^{28}$.

8. La thèse avancée ici est donc la suivante : ce qui s'exprime dans la lettre du 10 décembre 1513, avec l'hypothèse d'une dédicace à Giuliano, est un effet collatéral de la Constitution du 22 novembre 1513 décidée par Lorenzo. En recréant un ordre sénatorial étouffant tout principe démocratique, cette Constitution apportait une réponse négative à la prescription de politique intérieure dont était porteur Le Prince, notamment en son neuvième chapitre. Machiavel y avait significativement valorisé une figure historique controversée, celle du roi révolutionnaire Nabis de Sparte (mort en 192 av. è. c.), exemplaire de la résistance menée contre une puissance étrangère grâce au soutien des masses populaires et à la répression d'aristocraties factieuses (Pr. 9.19) ; or, d'après Tite-Live, Nabis s'était aussi distingué par une critique radicale de la République romaine en tant qu'elle était fondée sur un système censitaire établissant la prééminence $d^{\prime} u n$ ordre sénatorial séparé29. Si jamais Machiavel a effectivement tenté de remettre à Lorenzo la copie de dédicace - comme le suggère I'historiette selon laquelle le jeune homme se détourna de l'auteur du Prince pour recevoir plutôt un don de chiens - il n'a pu disposer que d'une fenêtre très étroite pour le faire, dans les jours qui suivirent l'échéance de ses condamnations. En tout état de cause, Machiavel ne

\footnotetext{
${ }^{28}$ Cf. J. Barthas, "Machiavel et la fonction tribunitienne ", in La représentation avant le gouvernement représentatif, éd. S. Hayat, C. Péneau et Y. Sintomer, Rennes, PUR, 2020, p. 265-87.

${ }^{29} \mathrm{Cf}$. éd. Pedullà, p. 112-13 note 17, et J. Barthas, "Cleomenes redivivus. Machiavelli from The Prince to the Discourses ", in Essays in Honour of Robert Black, éd. J. Davies et J. Monfasani, Leyde, Brill, 2021, p. 83106.
} 
pouvait plus le lui présenter après le 22 novembre 1513 ; tel que le texte nous est parvenu, il bornait étroitement les limites à l'intérieur desquelles l'ex-chancelier était prêt à se compromettre à Florence en tant que médicéen. Livre des républicains, Le Prince exprimait la position la plus avancée du moment pour tenter de sauver l'expérience populaire du Grand Conseil.

Jérémie Barthas

CNRS - IHMC (UMR 8066, Paris) 